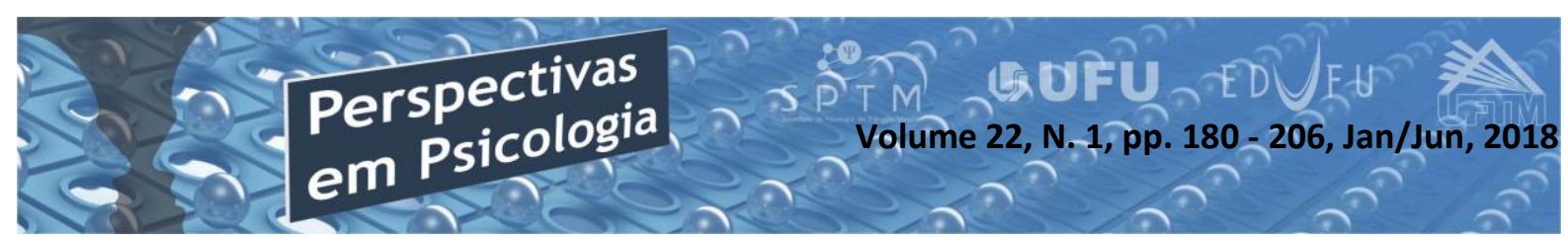

\title{
FATORES DE RISCO E DE PROTEÇÃO PARA EMITIR DELITOS VIOLENTOS: REVISÃO SISTEMÁTICA DA LITERATURA
}

\author{
André Vilela Komatsu \\ Marina Rezende Bazon \\ (Universidade de São Paulo (FFCLRP - Ribeirão Preto - SP)
}

\begin{abstract}
Resumo
Realizou-se uma revisão sistemática da literatura com objetivo de identificar os principais fatores de risco e de proteção à conduta violenta. 37 artigos foram selecionados/agrupados em cinco domínios. No domínio Indivíduo, destacam-se os constructos Baixa Empatia e Baixo Autocontrole. Na Família, os estudos ressaltaram aspectos de vivências estressoras como Abuso Físico e Rejeição Parental. Na Escola, a Expulsão e a Reprovação de Ano foram os aspectos mais relevantes. Quanto aos Pares, estes exercem forte influência no final da adolescência e início da vida adulta. O domínio Bairro/Comunidade não teve fatores com associação forte. Em relação aos fatores protetivos, as poucas variáveis investigadas estão no plano individual. Aponta-se a necessidade de estudos levando em consideração o fator cumulativo entre as variáveis.
\end{abstract}

Palavras-chave: violência; delinquência juvenil; fatores de risco; fatores de proteção; revisão sistemática.

\begin{abstract}
Risk and protective factors for violent offenses: a systematic review of the literature

A systematic review of the literature was carried out aimed to identify the main risk and protective factors for violent offense. 37 studies were selected/grouped in five domains. In the Individual domain, the Low Empathy and Low Self-Control constructs stand out. In the Family, the studies highlighted aspects of stressful experiences such as Physical Abuse and Parental Rejection. In the School, Expelled from school and Flunk were the most relevant aspects. Regarding the Peers, they exert a strong influence in late adolescence/early adulthood. The Neighborhood/Community domain had no strong association factors. Regarding the protective factors, the few variables investigated are at the individual level. It is pointed out the necessity of studies taking into account the cumulative factor among the variables.
\end{abstract}

Keywords: violence; juvenile delinquency; risk factors; protective factors; systematic review. 


\section{Introdução}

O Brasil é considerado um país com elevadas taxas de violência. Segundo o último levantamento do Atlas da Violência (Cerqueira et al., 2016, p. 6), mais de 59 mil pessoas morrem no país, por ano, vítimas de homicídio, o que significa 29,1 homicídios para cada 100 mil habitantes - taxa maior que a de homicídios em países em situação de guerra, como o Iraque, por exemplo. Neste cenário, os adolescentes ocupam um lugar de destaque por dois principais motivos. Primeiro porque a população jovem é a vítima preferencial dessa forma de violência. Entre os 15 e os 29 anos, a violência é a principal causa de morte, responsável por $39 \%$ dos óbitos dos indivíduos dessa faixa etária no Brasil (Waiselfisz, 2014, p. 27). Segundo porque atuam como agentes de violência. A título de exemplo, durante o ano de 2014, eles foram responsáveis por 3.951 homicídios, homicídios tentados ou latrocínio e por 12.196 roubos, roubos tentados ou lesão corporal (Fórum de Segurança Pública, 2017).

Esses dados são alarmantes tendo em vista os prejuízos sociais, econômicos e psicológicos infligidos às vítimas, às famílias e à sociedade. Ademais, o próprio envolvimento com a prática de atos violentos implica experiências negativas que podem impactar e comprometer o desenvolvimento psicossocial do jovem (Hirschfield, 2009), constituindo-se em fator de risco associado à manutenção desse ciclo de violências. Conforme pontuado por Assis, Deslandes e Santos (2005), "compreendem-se as diferentes formas de violência como graves obstáculos ao pleno desenvolvimento dos 35 milhões de adolescentes entre 10 e 19 anos no País (sejam as formas em que são vítimas, sejam as situações em que são os autores)".

Frente a esta problemática, pesquisadores têm buscado identificar os principais fatores associados à conduta infracional violenta. Lipsey e Derzon (1998) e Hawkings e colegas (2000) apresentam duas grandes revisões sobre os fatores de risco associados a crimes violentos e/ou graves. A síntese do conhecimento produzido até então identificou centenas de variáveis com algum efeito de medida associadas à conduta violenta. Não sendo possível trabalhar com cada uma delas separadamente, agruparam-nas em domínios: indivíduo, família, escola, pares e comunidade (Hawkings et al., 2000). Esse agrupamento foi útil por favorecer uma visão global dos aspectos mais problemáticos no plano pessoal e no dos contextos nos quais os indivíduos se desenvolvem, levando em consideração que, de forma reiterada, os estudos mostraram que a exposição a um ou a outro 
fator, isoladamente, pouco informa sobre o risco de um indivíduo cometer atos violentos, mas o acúmulo de diversos fatores para um mesmo indivíduo é bastante relevante para esta questão (Herrenkohl et al., 2000; Falk et al., 2014).

Outro ponto importante identificado por essas revisões é que os estudos indicavam que a maior parte dos crimes era realizada por um pequeno número de pessoas, o que para Lipsey e Derzon (1998) torna atraente a ideia de promover programas de prevenção com base nesses fatores, uma vez que, aplicados a uma população relativamente pequena, um programa eficaz tem potencial para evitar em boa medida o problema em questão. De fato, a ideia de que a massa dos crimes violentos é praticada por uma proporção diminuta de pessoas tem sido confirmada em estudos recentes, de caráter populacional, como o realizado por Falk e colegas (2014) na Suécia, no qual encontrou-se que $1 \%$ da população era responsável por $63 \%$ dos crimes violentos registrados. Nesse sentido, estudos econômicos indicam que para cada dólar investido em programas de prevenção, a sociedade economiza de sete a oito dólares em gastos futuros (Lee et al., 2012), sendo que tal economia pode ser tanto em relação ao tratamento das vítimas, quanto em relação às políticas de segurança pública, atinentes ao policiamento, aprisionamento e tratamento dos perpetradores. Assim, desenvolver e aplicar programas preventivos, alternativos, menos excludentes e mais eficazes, pode representar um grande avanço da sociedade na direção da redução da violência e da promoção do bem-estar individual e social.

As revisões de Lipsey e Dorzen (1998) e Hawkings e colegas (2000) produziram significativo conhecimento sobre os fatores de risco associados à infração crônica, grave e violenta, com claras implicações para políticas públicas sociais, de segurança e de saúde da criança e do adolescente. No entanto, ambas focalizam apenas os fatores de risco, sem destacar os fatores de proteção. Sabe-se que, para diversos desdobramentos psicológicos e comportamentais, a promoção de fatores de proteção é tão importante - se não mais - que a atenuação/eliminação dos fatores de risco (Robbé, Vogel \& Stam, 2012). Além disso, desde a publicação dessas revisões já se passaram mais de 15 anos. Naquela época, Borum (2000) sugeriu que havia um crescente interesse dos pesquisadores em estudar comportamentos violentos e, como a violência envolvendo jovens não amainou, parece importante empreender a uma nova organização e a síntese dos conhecimentos produzidos pelas pesquisas realizadas a partir desse período. 
Posto isso, o presente estudo tem como objetivo identificar, por meio de uma revisão sistemática da literatura, os principais fatores de risco e de proteção para crianças e adolescentes do sexo masculino associados à emissão de delitos violentos. Considerando que fatores de risco e de proteção, assim como os padrões de comportamentos antissocial, não são sempre os mesmos entre meninos e meninas, optou-se por selecionar estudos com amostras do sexo masculino, tendo em conta que a problemática tem maior incidência neste seguimento, sendo o próprio gênero masculino um fator de risco significativo para o envolvimento em delitos violentos (Falk et al., 2014).

\section{Os conceitos abordados}

Delitos violentos. Numa primeira instância, tem-se que a violência de natureza interpessoal, segundo a definição da Organização Mundial da Saúde (OMS; Krug et al., 2002), refere-se a um comportamento que implica "uso intencional de poder ou força física, real ou sob a forma de ameaça, contra si próprio, outra pessoa, um grupo oи uma comunidade, que resulte ou tenha grande possibilidade de resultar em lesão, morte, dano psicológico, deficiência de desenvolvimento ou privação" (p. 5). Assim, tendo por base esta conceituação, considera-se que alguns atos juridicamente tipificados como delitos se caracterizam pelo uso da violência interpessoal, privilegiando-se, neste estudo, especialmente o homicídio, o estupro, o roubo, a lesão corporal, a ameaça e o sequestro (Katsiyannis et al., 2012; Falk et al., 2014).

Fator de risco. Compreende condições ou variáveis que aumentam a probabilidade para um indivíduo de um desfecho negativo (Schenker \& Minayo, 2005); no caso em foco, aumentam a probabilidade de o indivíduo praticar delitos violentos, comparando-o a outra pessoa da população que não esteja exposta às mesmas condições ou variáveis.

Fator de proteção. Compreende condições ou variáveis que diminuem a probabilidade de um desfecho negativo para um individuo (Schenker \& Minayo, 2005); no caso em foco, diminuem a chance de o indivíduo praticar delitos violentos, comparando-o a outra pessoa da população que não esteja exposta às mesmas condições ou variáveis.

\section{Método}

Bases de Dados e Estratégia de Busca

A busca bibliográfica foi realizada em uma base de dados internacional multidisciplinar (Web Of Science), em uma 
base internacional do campo da Psicologia (PsycINFO), em uma base internacional de educação (ERIC) e em uma biblioteca multidisciplinar nacional (SciELO). Nas bases internacionais, foi utilizada a seguinte combinação de descritores: ("adolescen*" or "juvenile") and ("risk factor*" or "protective fator*") and ("offense" or "crime*") and ("violen*"). Na biblioteca SciELO foi utilizada a seguinte combinação: ("adolesc *" OR "juveni*") AND ("violen*" OR "agressiv*") AND ("delito*" OR "infraç*" OR "crime*"). As palavras-chave foram buscadas no tópico (título, resumo, palavras-chave) do artigo, optando-se por selecionar artigos a partir do ano de 2001.

\section{Critérios de exclusão}

As publicações obtidas pelo procedimento de busca foram selecionadas a partir da leitura dos títulos e resumos, sob os seguintes critérios de exclusão: (1) estudos de revisão; (2) estudos que não tinham como participantes adolescentes do sexo masculino; e (3) estudos que não investigavam a relação de variáveis independentes, como risco ou proteção, e a dependente, a emissão de delitos violentos.

Nesta etapa do processo, 1.098 artigos de um total de 1.157 foram excluídos. $\mathrm{Na}$ sequência, os artigos restantes foram lidos integralmente e seus conteúdos foram sintetizados em uma tabela contendo as seguintes informações: ano de publicação, nomes dos autores, país em que a pesquisa foi realizada, domínios aos quais as variáveis investigadas pertencem, características dos participantes da pesquisa, instrumentos utilizados, definição dada à variável dependente, variáveis controladas, fatores de risco e/ou fatores de proteção identificados, variáveis sem associação com a variável dependente e magnitude do efeito. Nesta etapa, outros 23 artigos foram excluídos porque se verificou que esses abarcavam sob o título de "delitos violentos" comportamentos outros, que não se encaixam na definição adotada por este estudo. As informações compiladas na tabela serviram de guia para a análise crítica do conjunto de produções levantado.

\section{Resultados}

Conforme o mencionado, a combinação das palavras-chave resultou em um total de 262 artigos na base Web of Science, 716 na PsycINFO, 114 na ERIC e 65 na SciELO. Após a leitura dos resumos, remoção dos artigos duplicados e leitura dos textos na íntegra, restaram 37 artigos.

Parte dos artigos recuperados foi publicada nos anos de 2001, 2005 e 2006, com uma publicação em cada ano. Os anos seguintes, 2008 e 2009, tiveram duas 
publicações cada. Posteriormente, as publicações seguiram a seguinte distribuição: $2011(\mathrm{n}=4), 2012(\mathrm{n}=6)$, $2013(n=2), 2014(n=9), 2015(n=4)$, $2016(n=2)$ e $2017(n=3)$. Os países que mais publicaram foram os Estados Unidos $(\mathrm{n}=17)$, Suécia $(\mathrm{n}=6)$, Inglaterra $(\mathrm{n}=3)$ e Holanda $(\mathrm{n}=2)$. Os demais, África do Sul, Alemanha, Bélgica, Bósnia, Brasil, Canadá, Coréia do Sul, Croácia, El Salvador, Espanha e Japão, tiveram uma publicação cada.

Seguindo a categorização proposta por Hawkings e colegas (2000), os fatores de risco identificados nas investigações revisadas foram classificados em cinco domínios, de acordo com sua natureza: fatores individuais, familiares, escolares, relativos aos pares de idade e ao bairro/comunidade de residência. Pelo fato do valor preditivo deles mudar de acordo com o momento em que se fazem presentes no curso do desenvolvimento de um indivíduo (DHHS, 2001, p. 47), os fatores de risco também foram classificados em termos de faixa etária, tendo em vista aquela em que cada qual teria impacto mais importante.

Em termos de conteúdo, para além do nome do constructo indicador de risco, os principais apontamentos na literatura revisada foram compilados e são apresentados a seguir, segundo os domínios de classificação.
No plano das características individuais, como mostra a tabela 1, o primeiro constructo que aparece, apontado em duas publicações é o fato de Experienciar Situações Adversas na infância aumenta a chance de cometer delito violento do tipo sexual. Souverein, Ward, Visser e Burton (2016) identificaram, entre adolescentes sul-africanos, que sofrer abuso ou violência física na adolescência aumenta o risco do indivíduo perpetrar um delito violento, comparado aos jovens que não foram expostos a tais fatores. No estudo de Drury e colegas (2017) identificou-se que um grande número de adolescentes infratores sexuais dos Estados Unidos sofreu maus-tratos do tipo físico, verbal, emocional, sexual ou negligência e/ou abandono durante a infância. Esses eventos estressores podem assumir forte impacto no desenvolvimento humano, especialmente na ausência de fatores protetivos, uma vez que as crianças, além de sofrerem os maustratos mencionados, podem não contar com o suporte parental, que no caso são a causa do problema.

Outro fator que apareceu em duas publicações recuperadas, é Atitudes Antissociais. Este, às vezes chamado de "neutralização" (Esbensen, Peterson, Taylor \& Freng, 2009), representa uma série de pensamentos e crenças que inibem o constrangimento moral referente às regras e normas estabelecidas socialmente e que, 


\section{SISTEMÁTICA DA LITERATURA}

por isso, favorecem o engajamento em comportamentos divergentes ou delituosos como, por exemplo, considerar que "não há problema de bater em alguém que ameaçou minha família". Neste sentido, Beyers, Loeber, Wikstrom e Stouthamer-Loeber (2001) encontraram que adolescentes com pontuações elevadas na Escala de Atitudes Antissociais tinham duas vezes mais chances de serem presos por cometimento de delitos violentos, se comparados aos adolescentes com baixa pontuação. $\mathrm{Na}$ mesma linha, Esbensen e colegas (2009) identificaram que adolescentes de escolas públicas dos Estados Unidos cujas atitudes eram favoráveis a comportamentos divergentes revelavam ter realizado delitos violentos mais frequentemente que seus colegas.

Ainda no plano individual, referido em dois estudos, destaca-se também o Abuso de Substâncias, condição psiquiátrica diagnosticada pelas consequências negativas à saúde e ao cumprimento dos deveres sociais que o uso de álcool e/ou outras drogas produz ao indivíduo. Andrade, Junior, Teixeira e Fonseca (2011), utilizando amostras de adolescentes infratores brasileiros, encontraram que os abusadores de álcool possuíam mais que o dobro de chance de cometer um delito violento que os não abusadores. Essa tendência já havia sido demonstrada por Johansson e Kempf-
Leonard (2009) que, em seu estudo, levantaram os dados de todos os adolescentes infratores que haviam passado pelo sistema de justiça do Texas, verificando que aqueles que faziam uso abusivo de substâncias eram detidos por violência com mais frequência que os demais adolescentes.

Outra variável individual destacada por dois dos artigos levantados refere-se à classe das Respostas Fisiológicas, nas quais atividades neuroquímicas são mensuradas, denotando-se associação entre condutas/comportamentos e certos marcadores neurofisiológicos do organismo. Muñoz, Frick, Kimonis e Aucoin (2008), utilizando registros de condutância da pele para a reatividade de adolescentes frente a estímulos negativos, encontraram que aqueles que tinham baixa excitação/reatividade revelavam mais delitos que os jovens com maior reatividade frente aos estímulos. De modo semelhante, Jennings, Piquero e Farrington (2013) mensuraram a frequência cardíaca em repouso (FCR) de uma amostra sob a presença de estímulos. Os participantes foram acompanhados dos 8 aos 48 anos, e aqueles com baixa FCR $(<65)$ foram detidos por delito violento $40 \%$ mais frequentemente que os avaliados com alta FCR (> 65). Os autores relacionam a baixa excitação/reatividade fisiológica frente a estímulos com o traço de personalidade 
denominado "busca por sensações estimulantes", numa medida acima do considerado "normal", e o consequente envolvimento em atividades de risco, dentre as quais as atividades infracionais violentas.

$\mathrm{Na}$ linha das variáveis disposicionais, Kalvin e Bierman (2017) identificaram que Problemas na Regulação Emocional no final da adolescência é um forte preditor de comportamentos delituosos, especialmente a negatividade emocional (tendência em sentir emoções ruins, como a raiva). A frequência em que essas sensações emocionais são sentidas também é importante, segundo o estudo de Shetgiri Boots, Lin e Cheng (2016) que identificou que adolescentes que são frequentemente invadidos por estressores emocionais envolvem-se mais frequentemente em delitos envolvendo arma de fogo. Embora os autores não discutam a natureza dessa relação, a literatura sobre o assunto é vasta (Birkley \& Eckhardt, 2015; Roberton, Daffern \& Bucks, 2012) e sugere que essa tendência em reagir negativamente a estímulos ambientais seja uma característica temperamental, que pode ser modelada pelo ambiente, e que favorece o uso de comportamentos violentos como recurso nas interações pessoais ou como forma de alcançar objetivos.

A Baixa Empatia, ou sentimento reduzido de culpa, foi citada em três artigos e também aparece como uma das características do temperamento individual considerada risco para a violência. A empatia é um importante recurso cognitivo para entender e contextualizar os sentimentos e estados mentais de outra pessoa, sendo que seu déficit compromete os mecanismos que ajudam a inibir comportamentos que podem ferir afetiva ou fisicamente outra pessoa (Díaz-Galván, Ostrosky-Shejet \& Romero-Rebollar, 2015). Olate, Salas-Wright e Vaughn (2012) encontraram em uma amostra de jovens que aqueles identificados com baixa empatia eram mais propensos a participarem de gangues e possuíam risco para utilizar armas como instrumento de agressão três vezes maior que os jovens identificados com alta empatia. Beyers et al (2001) e Esbensen e colegas (2009) já haviam obtido resultados semelhantes em relação ao que eles chamaram de "sentimento de culpa reduzido", relacionado a dados obtidos por meio de autorrelato de delitos violentos por parte dos adolescentes.

Outro aspecto atrelado ao temperamento, fortemente associado a comportamentos violentos e que apareceu em cinco artigos recuperados, é o Baixo Autocontrole. Wikström e Trieber (2007) indicam que o autocontrole é a capacidade de regulação do próprio comportamento, resultado da interação entre traços do 
indivíduo (suas capacidades executivas) e o ambiente no qual a pessoa está inserida. $\mathrm{O}$ autocontrole remete, portanto, ao que a pessoa faz frente a um estímulo ambiental, em favor de uma consequência, em detrimento de outra. Para Wikström e Trieber (2007), subjacente ao autocontrole estão as capacidades executivas, ou funções executivas, que são habilidades como controle atencional, controle inibitório, memória de trabalho e flexibilidade cognitiva que um indivíduo tem disponível para exercer o controle de seus comportamentos. Dessa forma, o termo autocontrole é uma medida reservada para avaliar se um indivíduo responde com sucesso, de acordo com sua moralidade, quando confrontado com situações conflitantes ou a tentações.

Nessa linha, Gover, Park, Tomsich e Jennings (2011) identificaram que jovens adultos que haviam praticado violência física contra parceira íntima possuíam escore baixo em escala de autocontrole. Com base em dados de uma amostra populacional, nos Estados Unidos, Larson, Vaughn, Salas-Wright e Delisi (2014), em seu turno, encontraram que o baixo autocontrole estava associado não só à violência contra parceira íntima, mas também a violências sexuais contra desconhecidos e também a violência física de forma geral. Muftic, Grubb, Bouffard e Maljevic (2014), por sua, estudando amostras de adolescentes escolares, encontraram que apenas $12 \%$ dos investigados haviam se envolvido em delitos violentos, sendo que aqueles com baixo autocontrole revelavam comportamentos violentos com mais frequência que os demais adolescentes. Controlando o efeito de outras variáveis potencialmente de risco, Moberg e colegas (2015), analisando o histórico de vida de mais de 48 mil pessoas, encontraram que aquelas que, ao final da adolescência e início da vida adulta, apresentavam dificuldades para controlar o sentimento de raiva tinham uma chance $72 \%$ maior de serem presos por delito violento que indivíduos que não tinham essas dificuldades. Estudando especificamente adolescentes infratores, Fox, Perez, Cass, Baglivio e Epps (2015), com base em dados de uma amostra nacional, dos Estados Unidos, detectaram uma chance duas vezes maior de um adolescente ser preso por cometimento de delito violento ao pontuar alto em uma escala de impulsividade (aspecto referente a um baixo autocontrole associado, especialmente, à capacidade de planejar antes de agir).

\section{O Histórico de Condutas}

Divergentes, aferido com dados obtidos por meio de autorrelato ou por meio de registros oficiais, refere-se também a um aspecto de natureza individual. Este foi citado por cinco artigos e é considerado um dos mais 
importantes preditores de comportamento violento futuro. Dentro disso, Bui, Farrington, Ueda e Hill (2014), destacam os denominados "comportamentos de risco". Em um estudo comparativo entre Japão e Estados Unidos, esses autores identificaram que jovens com envolvimento em atividades que implicavam em comportamentos de risco, como fazer coisas perigosas apenas por diversão, possuíam duas a quatro vezes mais chances de revelarem um delito violento que jovens que não apresentavam tais comportamentos de risco. Nesse espectro, os problemas de comportamentos, de forma geral, como comportamentos agressivos, enfrentamento de figuras de autoridade, indisciplina, etc., também parecem predizer envolvimento em delitos violentos. Ibabe, Arnoso e Elgorriaga (2014) encontraram correlação positiva e significativa entre problemas de comportamento fora de casa e violência perpetrada por adolescentes contra membros da própria família, ambos aferidos por escalas. Outra modalidade de conduta divergente seriam os próprios delitos violentos. Ter um histórico de delitos violentos é identificado na literatura como um dos preditores mais fortes de comportamento violento futuro (Piquero, Theobald \& Farrington, 2014). Dessa forma, adolescentes que cometem delitos violentos possuem chances maiores de repetir esse tipo de comportamento ainda na adolescência (Rengle, Jennings \& Maldonado-Molina, 2012) ou mesmo na vida adulta (Brook et al., 2011), quando comparados a adolescentes da mesma idade sem implicação em delitos violentos. 
Tabela 1. Fatores de risco associados ao Domínio Individual

\begin{tabular}{|c|c|c|c|c|}
\hline 0-6 anos & 7-9 anos & 10-12 anos & 13-15 anos & 16 ou mais \\
\hline $\begin{array}{l}\text { - Experiências } \\
\text { adversas }\end{array}$ & $\begin{array}{l}\text { - Histórico de } \\
\text { Condutas } \\
\text { Divergentes }\end{array}$ & $\begin{array}{l}\text { - Detenção por } \\
\text { delitos graves } \\
\text { - Perpetrar } \\
\text { violência fora da } \\
\text { família } \\
\text { - Problemas de } \\
\text { conduta } \\
\text { - Desregulação } \\
\text { emocional }\end{array}$ & $\begin{array}{l}\text { - Histórico de } \\
\text { Condutas } \\
\text { Divergentes } \\
\text { - Ausência de culpa } \\
\text { / baixa empatia } \\
\text { - Relação sexual } \\
\text { precoce (antes dos } \\
\text { 14) } \\
\text { - Ser vítima de } \\
\text { agressão física } \\
\text { - Baixo autocontrole } \\
\text { / manejo da raiva } \\
\text { - Detenção por } \\
\text { delitos graves } \\
\text { - Perpetrar violência } \\
\text { fora da família } \\
\text { - Baixo nível de } \\
\text { excitação/ Busca por } \\
\text { sensações } \\
\text { - Impulsividade } \\
\text { - Abuso de álcool e } \\
\text { drogas }\end{array}$ & $\begin{array}{l}\text { - Transtorno de } \\
\text { dependência de } \\
\text { drogas } \\
\text { - Atitude antissocial } \\
\text { - Ausência de culpa / } \\
\text { baixa empatia } \\
\text { - Baixo autocontrole / } \\
\text { manejo da raiva } \\
\text { - Transtorno de } \\
\text { dependência de álcool } \\
\text { - Detenção por delitos } \\
\text { graves } \\
\text { - Perpetrar violência } \\
\text { fora da família } \\
\text { - Baixo nível de } \\
\text { excitação / freq. card. } \\
\text { - Engajamento em } \\
\text { comportamentos de } \\
\text { risco } \\
\text { - Transtorno de } \\
\text { personalidade }\end{array}$ \\
\hline
\end{tabular}

Nota: em negrito foram destacados os fatores de risco considerados mais fortes (Razão de chances > 3).

Passando para o domínio familiar, cujos resultados resumem-se na tabela 2 , uma das variáveis mais estudadas é a Criminalidade Parental. Essa foi investigada em cinco estudos recuperados nesta revisão (Britvic, Urlic \& DefinisGojanovic, 2006; Silva, Larm, Vitaro, Tremblay \& Hodgins, 2012; Falk et al., 2014; Kendler et al., 2014; Weijer, Jong, Bijleveld, Blokland \& Raine, 2017), sendo que todos indicam que o fato de um dos responsáveis pelo adolescente ter sido preso constitui um importante indicador de risco em relação ao envolvimento do(s) filho(s) em delitos violentos. Kendler e colegas (2014), em estudo com crianças adotadas na Suécia, encontraram que o fato de o pai adotivo (responsável, em contato direto com a criança) e o do pai biológico (sem contato direto com a criança) serem presos por crime violento, aumentava a chance do filho também ser preso, no futuro, em 
comparação a outros jovens cujos pais adotivos ou biológicos - não tinham histórico de detenção.

$\mathrm{O}$ último aspecto atinente à dimensão familiar são as Experiências Estressantes, como rejeições, abusos e conflitos entre os pais. Este tema apareceu em oito artigos. Britvic, Urlic e DefinisGojanovic (2006) focalizaram fatores nesse contexto e verificaram que a rejeição parental seria o mais forte fator de risco para a violência no âmbito familiar. Nofziger e Kurtz (2005), Britvic, Urlic, DefinisGojanovic (2006), Gover e colegas (2011) e Lahlah, Knaap e Bogaerts (2013), por sua vez, encontraram que sofrer abuso físico, ou punição física exagerada, pode representar um risco duas vezes maior para $\mathrm{o}$ envolvimento em delitos violentos. Essa relação não foi encontrada em relação ao abuso sexual (Nofziger \& Kurtz, 2005; Delisi et al., 2014; Fox et al., 2015). Além dessas situações atinentes à relação cuidador-criança/adolescente, outras experiências estressantes na família podem propiciar comportamentos violentos. Khan e Cooke (2008) encontraram que presenciar situações de violência entre os pais favorece o comportamento violento entre irmãos e, nesta mesma linha, Gover e colegas (2011) indicam que testemunhar violência mãe-pai aumenta o risco em mais de duas vezes para perpetrar violência contra o(a) parceiro(a) íntimo(a).

Tabela 2. Fatores de risco associados ao Domínio familiar

\begin{tabular}{lllll}
\hline 0-6 anos & 7-9 anos & $\mathbf{1 0 - 1 2}$ anos & 13-15 anos & 16 ou mais \\
\hline- & - Criminalidade & - Criminalidade & - Rejeição & - Testemunhar \\
Criminalidade & parental & parental & parental & violência parental \\
parental & - Estresse e & - Estresse e & - Responsáveis & \\
& conflito familiar & conflito familiar & punir fisicamente & \\
& - Testemunhar & - Estresse e & - Criminalidade & \\
& de violência & conflito familiar & parental & \\
& parental & - Testemunhar & - Testemunhar & \\
& - Irmão cometer & violência & violência parental & \\
& crime & parental & & \\
& & - Irmão cometer & & \\
& & crime & & \\
& & &
\end{tabular}

Nota: em negrito foram destacados os fatores de risco considerados mais fortes (Razão de chances > 3).

No domínio escola, encontram-se indicações sobre as variáveis Reprovação e
Expulsão da escola, mencionados em dois artigos, como mostra a tabela 3. Katsiyannis 
e colegas (2012), com base em dados obtidos junto a amostras populacionais de escolas públicas e privadas dos Estados Unidos, identificaram que estudantes que haviam reprovado o ensino médio e estudantes que haviam sido expulsos da escola possuíam uma chance 3,4 e 5,4 maior que seus pares, que não haviam vivido essas situações negativas em relação à escola, de se envolverem em delitos violentos. Em El Salvador, Olate, Salas-Wright e Vaughn (2012) obtiveram resultado semelhante. Verificaram que adolescentes expulsos da escola possuíam risco 5,9 vezes maior de se envolver em atos violentos, se comparados a adolescentes que não tinham a mesma experiência.

Outro importante fator de risco identificado em âmbito escolar é a Agressão Física ou Verbal a Professores, citada por um artigo. Mensuradas por um questionário de agressividade cujas respostas eram contabilizadas por uma escala likert de cinco pontos, Khan e Cooke (2008) identificaram que a agressão a professores, física ou verbal, é um forte preditor para perpetrar violência física grave, com uso de armas potencialmente letais, contra os próprios irmãos. Esse resultado foi encontrando junto a uma amostra de adolescentes em conflito com a lei, o que significa que essa variável distingue adolescentes infratores violentos dos não violentos.

Finalizando o domínio escolar, as variáveis Ambiente Escolar Negativo, referente a escolas inseguras e desorganizadas, e Atitudes Negativas Frente à Escola, caracterizada por crenças negativas em relação aos estudos e aos professores como, por exemplo, achar que as tarefas escolares são uma perda de tempo, foram citadas por um e dois artigos, respectivamente. Esbensen e colegas (2009) encontraram que a prática de atos violentos é favorecida em escolas cujo ambiente se mostra desorganizado e criminalizado, enquanto Pardini e colegas (2012) e Souverein e colegas (2016) identificaram que adolescentes descrentes na escola e na educação como oportunidade de um futuro melhor praticavam mais atos violentos que os adolescentes que tinham atitudes positivas frente à escola e à educação. 
Tabela 3. Fatores de risco associados ao Domínio escolar

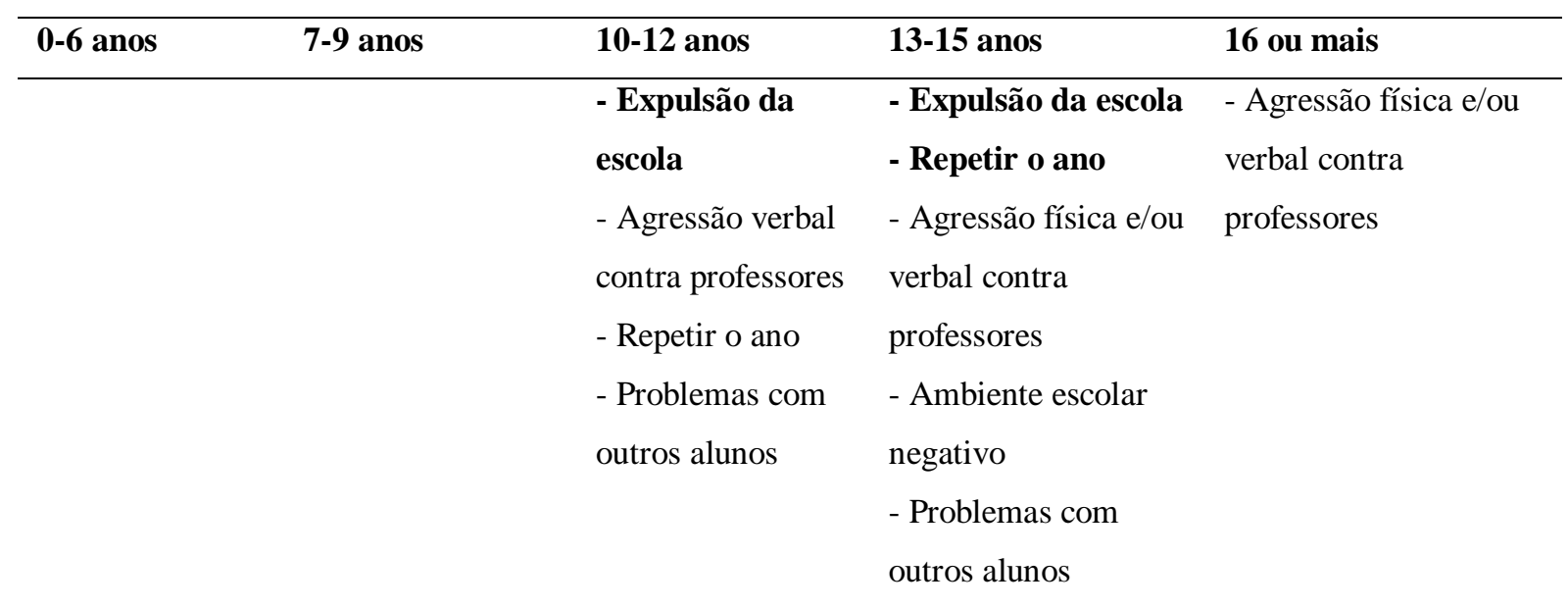

Nota: em negrito foram destacados os fatores de risco considerados mais fortes (Razão de chances > 3).

Passando para o domínio dos pares de idade, a tabela 4 mostra que há uma forte associação entre o envolvimento com delitos violentos e Número de Pares Infratores (três artigos), Pares Infratores Violentos (um artigo) e Pertencimento a Gangues (dois artigo). Beyers e colegas (2001), Esbensen e colegas (2009) e Olate, Salas-Wright e Vaughn (2012), por exemplo, utilizando métodos distintos, identificaram forte associação entre número de pares infratores e emissão de delitos violentos. Nofziger e Kurtz (2005), em estudo transversal retrospectivo, encontraram que frequentar pares que praticam atos violentos aumenta o risco para o autorrelato de infrações violentas. E Johansson e Kempf-Leonard (2009) identificaram, em meio a adolescentes infratores, que a pertencer a gangues elevava o risco de participação em crimes violentos em 1,5 vezes.

Tabela 4. Fatores de risco associados ao Domínio dos pares

\begin{tabular}{llll}
\hline 0-6 anos & 7-9 anos & $10-12$ anos & 16 ou mais \\
\hline & $-\mathrm{N}^{\circ}$ de pares & $-\mathrm{N}^{\circ}$ de Pares \\
& infratores & infratores \\
& - Engajamento com & - Engajamento com \\
& pares que praticam & pares que praticam \\
& violência & violência \\
& - Envolvimento em & \\
\hline
\end{tabular}

Nota: em negrito foram destacados os fatores de risco considerados mais fortes (Razão de chances > 3). 
Por último, a tabela 5 mostra as variáveis de risco pertencentes ao domínio bairro/comunidade. Muftic e colegas (2014) identificaram que residir em um Bairro Desorganizado e Criminalizado pode favorecer o surgimento de atos violentos. Um bairro degradado, percebido como perigoso devido às taxas de criminalidade, e devido à existência de pouco aparato social, associa-se ao aumento do relato de envolvimento em delitos violentos. Nofziger e Kurtz (2005), por sua vez, já haviam encontrado que adolescentes que testemunham violência no cotidiano de suas comunidades, como brigas, roubos, venda de drogas e outros tipos de crime, possuem maior risco de se envolver em comportamentos violentos. Somado a isso, Boutwell e colegas (2017), em um estudo demográfico/ecológico, identificaram que a exposição à substância chumbo aumenta significativamente as taxas de criminalidade violenta na região. Pode-se pensar que tal constatação deve-se ao fato do chumbo ser mais comumente encontrado em bairros pobres (e atribuir essas taxas à condição socioeconômica), mas sabe-se que o chumbo é uma substância tóxica para vários órgãos humanos, especialmente $\mathrm{o}$ cérebro, o que estaria ligado a várias disfunções (Reyes, 2007).

Tabela 5. Fatores de risco associados ao Domínio Comunitário

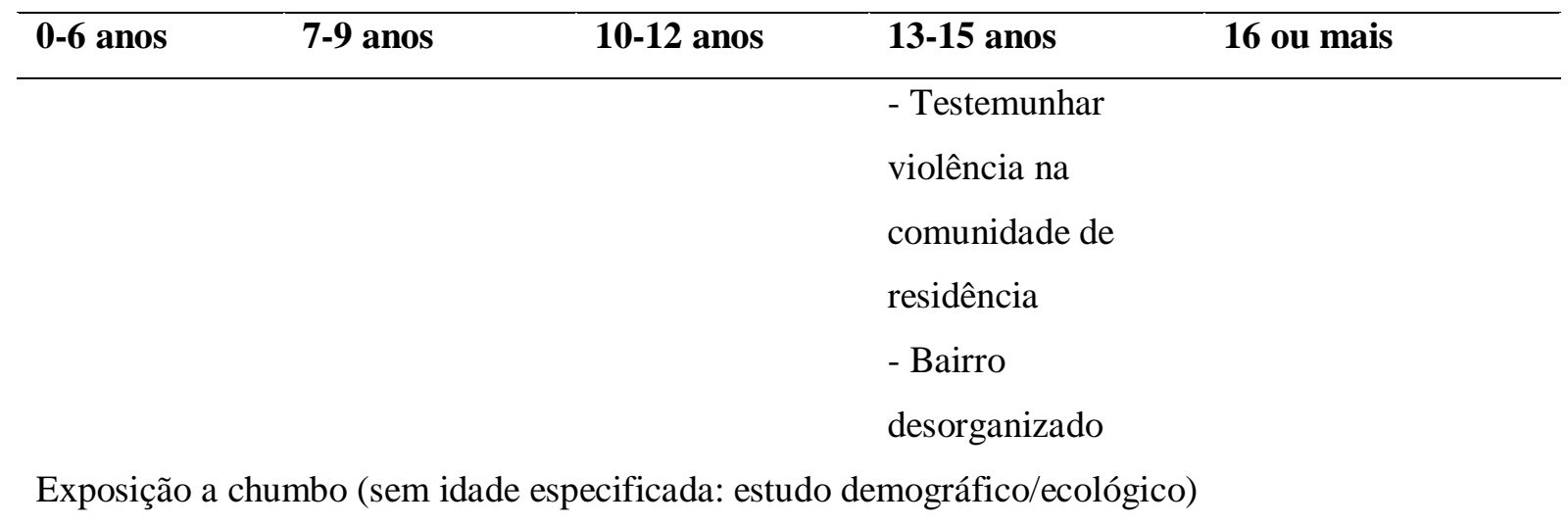

Nota: em negrito foram destacados os fatores de risco considerados mais fortes (Razão de chances > 3).

Quanto aos fatores de proteção, os dados encontrados, referentes ás publicações recuperadas, são sintetizados na Tabela 2, também agrupados por domínios. Devido ao baixo número de fatores de proteção identificados, decidiu-se por não os identificar por faixa etária, até porque não havia referência clara a este respeito nos estudos.

No domínio das características individuais, as duas variáveis que mais se destacam devido ao peso do efeito protetivo que parecem exercer são o Autocontrole e 
a Idade do Primeiro Delito Tardia. Essas são tratadas respectivamente em quatro e um artigos. $\mathrm{O}$ autocontrole, às vezes descrito como "planejar antes de agir" (Fox et al., 2015) ou como "saber manejar o sentimento de raiva" (Moberg et al., 2015), conforme o já mencionado, exerce um importante papel no controle de comportamentos violentos. Dessa forma, em uma perspectiva dicotômica de "baixo e alto autocontrole", indivíduos com pontuações elevadas em escalas que avaliam esse constructo têm chances menores de praticar delitos violentos, quando comparados a indivíduos com pontuações baixas (Muftic et al., 2014; Larson et al, 2014; Fox et al., 2015; Moberg et al., 2015). Em relação à idade do primeiro delito, seja ele violento ou não, quanto mais tarde for esta, menos provável o envolvimento em futuros atos violentos (Fox et al., 2015).

Outro aspecto do indivíduo identificado por um artigo como fator protetivo são as Habilidades Verbais, juntamente com o traço de personalidade "Baixa" Frieza (sendo o Traço Frieza associado a pouco sentimento de culpa). Muñoz e colegas (2008), trabalhando especificamente com adolescente em conflito com a lei, identificaram que aqueles com maior repertório de habilidades verbais e, ao mesmo tempo, com baixa pontuação na escala de Frieza do
Inventory of Callous-Unemotional Traits tinham menos chances de terem se envolvido em prática de delitos violentos que aqueles que pontuaram mais em uma ou outra dessas variáveis.

No domínio da família, o único fator que apareceu foi o Vínculo aos pais no início da adolescência. Este constructo, referente ao tempo e ao modo como o jovem passa o tempo com seus pais ou cuidadores, reduziria em quase a metade as chances de um adolescente emitir delitos violentos quando comparado aos adolescentes que teriam vínculo fragilizado (Mufitic et al., 2014).

$\mathrm{Na}$ escola, os dois fatores protetivos destacados foram Bom Desempenho Acadêmico e Apego à Escola, citados por um artigo cada. O desempenho acadêmico é o sucesso acadêmico, geralmente indicado pelo fato de tirar notas boas e progredir de ano. Um desempenho acadêmico positivo reduz a chance de envolvimento em delitos violentos em 44\% (Pardini et al., 2012). O apego à escola indica o grau de proximidade e o afeto que o adolescente sente com relação à escola e às autoridades escolares, sendo que um apego alto reduz em $12 \%$ as chances dele se envolver em violência, em comparação a adolescentes com baixo apego escolar (Mufitic et al., 2014).

Por último, possuir Poucos Pares Infratores, ou seja, ter uma rede de relacionamento em poucas pessoas realizam 
atos delituosos, é considerado um importante fator protetivo, reduzindo em $60 \%$ a em $70 \%$ as chances de envolvimento em delitos aos 13-14 anos e 15-18 anos, respectivamente (Pardini et al., 2012).

Tabela 6. Fatores de proteção para a emissão de delitos violentos

\begin{tabular}{ll}
\hline Domínio & Fator protetivo \\
\hline Indivíduo & - Autocontrole / baixa impulsividade / manejo da raiva \\
& - Idade do primeiro delito tardia \\
& - Alta habilidade verbal combinada com baixa pontuação na escala de frieza \\
Família & - Vínculo parental \\
& \\
Escola & - Bom desempenho acadêmico \\
& - Apego à escola \\
Pares & \\
Bairro/comunidade &
\end{tabular}

\section{Discussão}

A partir da sistemática adotada, pode-se afirmar, de modo reduzido, que as diversas investigações analisadas colocaram o domínio Indivíduo em destaque, uma vez que esse concentra a maior quantidade de fatores de risco estudados e identificados como relevantes, sublinhando-se a importância, nesse plano, dos constructos Baixa Empatia e Baixo Autocontrole. No domínio Família, encontrou-se que os fatores de risco podem estar presentes desde antes do nascimento até o final da adolescência, ressaltando aspectos de vivências estressoras como
Abuso Físico e Rejeição Parental. Na Escola, os fatores de risco são relevantes a partir do final da infância, perdendo força no final da adolescência, sendo a Expulsão e a Reprovação de Ano os aspectos mais importantes. Com relação aos Pares, os fatores de risco exercem forte influência no final da adolescência e início da vida adulta. Com menor destaque, o domínio Bairro/Comunidade foi composto por dois fatores, mas nenhum com associação forte. A classificação dos fatores de risco em domínios, como o aqui apresentado, deve ser visto como um recurso didático para organizar as informações no campo, sobre as variáveis identificadas. No mundo 
real esses domínios são inseparáveis no tocante à interinfluência que existe entre as diferentes variáveis, nos diferentes domínios, sendo que os programas de prevenção da violência devem articular todas essas esferas. Assim, os fatores de risco, analisados isoladamente, não são suficientes para explicar o comportamento violento. Esbensen e colegas (2009) identificaram um ponto de corte ótimo de sete fatores de risco cumulativos para o risco de violência ser considerado, enquanto Herrenkohl e colegas (2000) encontraram que a chance de uma pessoa cometer algum delito violento aos 18 anos seria dez vezes maior para crianças expostas a mais de cinco fatores de risco aos 10 anos idade, se comparadas a crianças expostas a menos de dois fatores de risco na mesma idade. Assim, deve-se interpretar que é o acúmulo de fatores de risco que é responsável por determinar o grau de risco associado à manifestação de atos violentos a que um indivíduo está submetido, e não a simples exposição a um ou a outro.

Ademais, conforme apontado por Farrington (2000), um dos maiores desafios em identificar fatores de risco é distinguir o que é causa e o que é apenas correlato das causas do comportamento violento. Ser expulso da escola, por exemplo, não é necessariamente um fator causal da emissão de delitos violento, mas é um dos fatores com maior poder de predição encontrado no presente estudo. Uma das possíveis explicações para essa forte associação é que a expulsão escolar é uma das últimas medidas tomadas pela escola em relação a um jovem que provavelmente já vem apresentando significativos problemas de comportamento, no plano do relacionamento com alunos e professores, no de assiduidade às aulas, assim como de desempenho escolar. Este é, portanto, mais propriamente um indicador de risco, apontando para a existência de uma série de problemas acumulados e de dificuldades enfrentadas pelo adolescente. Para reduzir o risco de violência de um jovem nessas condições não basta fazer com que ele não seja expulso, mantendo-o na escola $o$ máximo de tempo possível. A prevenção ou a intervenção, neste caso, seria evitar que as adversidades escolares se acumulassem a ponto de se cogitar sua expulsão da escola. Evitar o acúmulo desses problemas significa não apenas ajudar o adolescente a ajustar-se ao ambiente escolar, mas também preparar a escola para receber crianças e adolescentes com mais dificuldades, ouvilos e dialogar com a família para buscar entender possíveis situações conflituosas e manejá-las adequadamente.

Analisando a faixa etária na qual incidem os fatores de risco, percebe-se que os fatores escolares e familiares perdem força no final da adolescência, momento em que fatores individuais e os relacionados às 
características dos pares se destacam. Bazon, Silva e Ferrari (2013) atribuem essa mudança aos processos próprios da adolescência, como a busca por liberdade e por autonomia, juntamente com o anseio de ser aceito pelo grupo de pares. Os autores pontuam que a família não perde sua função socializadora, na adolescência, mas as relações estabelecidas fora do reduto familiar, nesse período da vida, passam a ocupar uma posição de destaque. De todo modo, a constatação de que existem fatores específicos que podem atuar em uma fase, mas não em outra, nos leva a concluir que os programas de prevenção devem ser direcionados a faixas etárias específicas. $\mathrm{Na}$ linha do que aponta a Organização das Nações Unidas (2015), intervenções de prevenção e de tratamento específicos aos diferentes estágios podem produzir resultados mais significativos no combate à violência, lidando com a noção de períodos sensíveis, que remetem a períodos limitados de tempo, nos quais a pessoa se mostra muito mais sensível às influências do ambiente, o que acaba por interferir intensamente em seu desenvolvimento, facilitando a aprendizagem de habilidades ou o desenvolvimento de aptidões e competências.

Deve-se destacar que características individuais como baixo autocontrole e ausência de culpa / baixa empatia se destacam como fatores de risco, em geral, do meio ao final da adolescência, sendo que indivíduos pontuando baixo em autocontrole e em empatia possuem chances mais de três vezes maiores de serem detidos por delito violento, ao ingressarem na vida adulta, se comparados a indivíduos que possuem alta pontuação nessas variáveis (Larson et al., 2015; Olate, Salas-Wright \& Vaughn, 2012). O baixo autocontrole tem sido associado a diversos desdobramentos negativos na vida das pessoas (Shoda, Mischel \& Peake, 1990; Eigsti et al., 2006), visto que a convivência em sociedade frequentemente exige $o$ controle de impulsos e o adiamento da satisfação de necessidades imediatas. Em relação à empatia, os estudos têm indicado seu importante papel na inibição de comportamentos agressivos, pois quando o indivíduo é capaz de perceber as sensações e as emoções do outro, o comportamento agressivo se torna mais custoso (DíazGalván, Ostrosky-Shejet \& RomeroRebollar, 2015). É interessante notar, no tocante à associação entre a baixa empatia e emissão de comportamentos violentos, na adolescência, que os circuitos neuronais associados a ambos os comportamentos se sobrepõem no cérebro (Moya-Albiol, Herrero \& Bernal, 2010).

Todavia, é preciso ressaltar que o desenvolvimento tanto do autocontrole quanto da empatia se dá nas interações sociais e relacionais (Tarullo, Obradovic, 
Gunnar, 2009; Knafo, Zahn-Waxler, Van Hulle, Robinson, 2008). Nesse ponto, é importante observar que a família, a escola e o entorno comunitário são instituições responsáveis pela socialização dos jovens, de modo que, se alguns adolescentes apresentam baixo autocontrole e baixa empatia, pode-se dizer que as instituições envolvidas em seu processo de socialização estão, de algum modo, falhando em suas funções.

Em relação aos fatores protetivos, apesar dos indicativos de que a promoção da resiliência pode ser mais eficaz que a remoção/diminuição dos riscos (Robbé, Vogel \& Stam, 2012), nota-se uma clara preferência dos pesquisadores em focar os fatores de risco. Nesse plano, contudo, de acordo com a revisão realizada, destacaramse também as variáveis no domínio Indivíduo. Percebe-se que alguns dos fatores de proteção são exatamente o contraponto de fatores de risco, tal como autocontrole (vs. baixo autocontrole) ou apego aos pais (vs. pouco apego aos pais), ao passo que outras variáveis como, por exemplo, habilidade verbal, parecem funcionar como fator protetivo, embora sua ausência não seja necessariamente um fator de risco específico ao envolvimento dos adolescentes em práticas de atos violentos. Nesse sentido, Tremblay (1999) aponta que aprender a usar a linguagem para se relacionar e satisfazer às próprias necessidades é um dos mais importantes fatores de proteção contra a violência física, embora obter um baixo desenvolvimento da linguagem não significa que a violência será usada como prática alternativa.

Nota-se que no domínio Bairro/Comunidade nenhum fator de proteção foi identificado. Não é possível dizer que não há fatores comunitários que possam proteger os jovens de envolveremse em atos violentos; apenas não foram recuperados estudos que se dedicaram a estudar este domínio. Dessa forma, mostrase importante a implementação de mais estudos nesta direção, a exemplo dos que foram realizados em relação à delinquência em geral (não necessariamente violenta), identificando-se fatores protetivos gerais nesse plano como, por exemplo, suporte/apoio social da parte dos vizinhos, da coletividade, e controle comunitário (Jain \& Cohen, 2012).

Por fim, é possível observar que as publicações, oriundas em sua maioria dos Estados Unidos e da Suécia e, em seguida, da Inglaterra, atrelam-se à existência de uma sólida agenda de pesquisa, bem coordenada entre a academia e as instituições governamentais. A maioria dos estudos estadunidenses vem de quatro grandes projetos de pesquisa com amostras populacionais: o Denver Youth Survey, o Pittsburgh Youth Study, o Rochester Youth Development Study e o Seattle Social 
Development Program. Na Suécia, as investigações apóiam-se, em geral, em dados oriundos de um sistema de registros de informações de todos os habitantes do país, de modo que lá se consegue articular com facilidade dados de registro civil, de cuidados médicos, criminais, entre outros, de modo a organizar informações anônimas sobre toda a população e produzir conhecimento específico, em diferentes áreas. Na Inglaterra, um estudo longitudinal de 42 anos (o Cambridge Study in Delinquent Development) é a fonte das informações por detrás das publicações recuperadas. Tendo sido financiada com verba pública, essa forneceu e ainda fornece subsídios fundamentais às políticas públicas na área, neste país.

\section{Considerações Finais}

A presente revisão tem o mérito de explorar fatores de risco e de proteção, e não apenas os fatores de risco, como em revisões anteriormente realizadas. Outro aspecto positivo é o de ter se esmerado em focalizar estudos que tratassem de fatores específicos ao envolvimento dos adolescentes em delitos violentos, conforme definição da OMS (2002), não tratando indiscriminadamente 0 envolvimento em atos infracionais.
Distinguiu-se, assim, os comportamentos referentes à delinquência crônica (repetitiva) e à delinquência grave (violenta). Ademais, são positivos também os fatos de se ter dado destaque às variáveis representando maior risco, com base em medida de magnitude, e às etapas do desenvolvimento onde esses parecem impactar com maior força, o que pode auxiliar futuras pesquisas e sugerir pistas para a preparação de possíveis programas de prevenção. Contudo, é preciso levar em conta que a síntese da literatura aqui apresentada não considera os efeitos cumulativos de diversos fatores de risco, no tempo, nem os contrabalanceia com os diferentes fatores de proteção. É preciso que novas pesquisas explorem os efeitos dos fatores protetivos em diferentes faixas etárias, contextos e populações.

No Brasil, a exemplo dos países que mais produzem conhecimento neste campo, governo e academia deveriam atuar em parceria, de modo a produzir conhecimento confiável e de qualidade na área; caso contrário, as decisões e as ações nesse campo, em nosso contexto, terão sempre por base informações imprecisas, tendendo a ser guiadas mais propriamente por preconcepções e pressões sociais baseadas em diferentes interesses. 


\section{Referências}

Andrade, R. C., Junior, F. A., Teixeira, I. A., \& Fonseca, V. A. D. (2011). Prevalência de transtornos psiquiátricos em jovens infratores na cidade do Rio de Janeiro (RJ, Brasil): estudo de gênero e relação com a gravidade do delito. Ciência e Saúde Coletiva, Vol. 16(4), pp. 2179-2188. DOI 10.1590/S1413-81232011000400017.

Assis, S.G., Deslandes, S.F., \& Souza, N.C. (2005). Violêcia na adolescência: sementes e frutos de uma sociedade desigual. In: SOUZA, E. R.; MINAYO, M. C. S. (Org.). Impacto da violência na saúde dos brasileiros. Brasília: Ministério da Saúde, 2005. p. 09-33.

Bazon, M. R., Silva, J. L., \& Ferrari, R. M.. (2013). Trajetórias escolares de adolescentes em conflito com a lei. Educação em Revista, 29(2), 175-199. DOI 10.1590/S010246982013000200008 .

Beyers, J. M., Loeber, R., Wikstrom, P-O. H., \& Stouthamer-Loeber, M. (2001). What predicts adolescent violence in better-off neighborhoods? Journal of Abnormal Child Psychology, Vol. 29, No. 5, 2001, pp. 369-381. DOI 10.1023/A:1010491218273.

Birkley, E. L., \& Eckhardt, C. I. (2015). Anger, hostility, internalizing negative emotions, and intimate partner violence perpetration: A meta-analytic review. Clinical Psychology Review, 37, 40-56.DOI 10.1016/j.cpr.2015.01.002

Borum, R. (2000). Assessing Violence Risk among Youth. Journal of Clinical Psychology, 56, 1263-1288. DOI 10.1002/1097-4679(200010)56:10<1263::AID-JCLP3>3.0.CO;2-D

Boutwell, B. B., Nelson, E. J., Qian, Z., Vaughn, M. G., Wright, J. P., Beaver, K. M., . . . Rosenfeld, R. (2017). Aggregate-level lead exposure, gun violence, homicide, and rape. Plos One, 12(11). DOI 10.1371/journal.pone.0187953.

Britvić, D., Urlić, I., Definis-Gojanović, M. (2006). Juvenile perpetrators of homicides and attempted homicides--a case control study. Coll. Antropol. 30 (2006) 1: 143-147.

Brook, D. W., Brook, J. S., Rubenstone, E., Zhang, C., \& Saar, N. S. (2011). Developmental Associations Between Externalizing Behaviors, Peer Delinquency, Drug Use, Perceived Neighborhood Crime, and Violent Behavior in Urban Communities. Agressive Behavior. Volume 37, pp. 349-361. DOI 10.1002/ab.20397.

Bui, L., Farrington, D. P., Ueda, M., Hill, K. G. (2014). Prevalence and Risk Factors for SelfReported Violence of Osaka and Seattle Male Youths. International Journal of Offender Therapy and Comparative Criminology, Vol. 58(12), pp. 1540-1557. DOI $10.1177 / 0306624 X 13501472$.

Cerqueira, D., Ferreira, H., Lima, R. S., Bueno, S., Hanshiro, O., Batista, F., \& Nicolato, P. (2016). Atlas da Violência 2016. Brasília: IPEA e FBSP.

Delisi, M., Vaughn, M. G., \& Caudill, J. W. (2014). Does Childhood Sexual Abuse Victimization Translate Into Juvenile Sexual Offending? New Evidence. Violence and Victims. DOI 10.1891/0886-6708.VV-D-13-00003. 
Department of Health and Human Services (DHHS). (2001). Youth violence: a report of the Surgeon General.

Díaz-Galván, K. X., Ostrosky-Shejet, F. \& Romero-Rebollar, C. (2015). Cognitive and affective empathy: The role in violent behavior and psychopathy. Rev Med Hosp Gen Méx. 78(1):27-35. DOI 10.1016/j.hgmx.2015.03.006.

Drury, A., Heinrichs, T., Elbert, M., Tahja, K., Delisi, M., \& Caropreso, D. (2017). Adverse childhood experiences, paraphilias, and serious criminal violence among federal sex offenders. Journal of Criminal Psychology, 7(2), 105-119. DOI 10.1108/JCP-11-20160039.

Eigsti, I. M., Zayas, V., Mischel, W., Shoda, Y., Ayduk, O., Dadlani, M.B., Davidson, M.C., Lawrence Aber, J., \& Casey, B. J. (2006). Predicting cognitive control from preschool to late adolescence and young adulthood. Psychological Science, Jun, 17(6), pp. 478-84. DOI 10.1111/j.1467-9280.2006.01732.x.

Esbensen, F., Peterson, D., Taylor, T. J., \& Freng, A. (2009). Similarities and Differences in Risk Factors for Violent Offending and Gang Membership. The Australian and New Zealand Journal of Criminology, Vol. 42, No. 3. pp. 310-335. DOI 10.1375/acri.42.3.310.

Falk, O., Wallinius, M., Lundstrom, S., Frisell, T., Anckarsater, H., Kerekes, N. (2014). The 1 $\%$ of the population accountable for $63 \%$ of all violent crime convictions. Soc Psychiatry Psychiatr Epidemiol, 49:559-571. doi: 10.1007/s00127-013-0783-y. DOI 10.1007/s00127-013-0783-y.

Farrington, D. P. (2000). Psychosocial predictors of adult antisocial personality and adult convictions. Behavioral Sciences \& the Law Special Issue: International Perspectives of Psychopathic Disorders, Volume 18, Issue 5, pages 605-622. DOI 10.1002/10990798(200010)18:5<605::AID-BSL406>3.0.CO;2-0.

Fox, B. H., Perez, N., Cass, E., Baglivio, M. T., \& Epps, N. (2015). Trauma changes everything: Examining the relationship between adverse childhood experiences and serious, violent and chronic juvenile offenders. Child Abuse \& Neglect, 46, pp. 163-173. DOI 10.1016/j.chiabu.2015.01.011.

Fórum Brasileiro de Segurança Pública. (2013). Anuário Brasileiro de Segurança Pública. ISSN 1983-7364, ano 7.

Gover, A. R., Park, M., Tomsich, E. A., \& Jennings, W. G. (2011). Dating Violence Perpetration and Victimization Among South Korean College Students: A Focus on Gender and Childhood Maltreatment. Journal of Interpersonal Violence, 26(6), pp. 12321263. DOI 10.1177/0886260510368161.

Grieger, L., \& Hosser, D. (2012). Attention deficit hyperactivity disorder does not predict criminal recidivism in young adult offenders: Results from a prospective study. International Journal of Law and Psychiatry, vol. 35, pp. 27-34. DOI 10.1016/j.ijlp.2011.11.005. 
Herrenkohl, T. L., Maguin, E., Hill, K. G., Hawkins, J. D., Abbott, R. D., \& Catalano, R. F. (2000). Developmental risk factors for youth violence. Journal of Adolescent Health, 6:176-186. DOI 10.1016/S1054-139X(99)00065-8.

Hawkins, D. J., Herrenkohl, T. I., Farrington, D. P., Brewer, D., Catalano, R. F., Harachi, T. W., \& Cothern, L. (2000). Predictors of Youth Violence. Washington, DC: Office of Juvenile Justice and Delinquency Prevention - Juvenile Justice Bulletin. DOI 10.1037/e524202006-001.

Hirschfield, P. (2009). Another way out: the impact of juvenile arrests on high school dropout. Sociology of Education, 82 (4), 368-393. DOI 10.1177/003804070908200404.

Ibabe, I., Arnoso, A., \& Elgorriaga, E. (2014). Behavioral problems and depressive symptomatology as predictors of child-toparent violence. The European Journal of Psychology Applied to Legal Context, vol. 6, pp. 53-61. DOI 10.1016/j.ejpal.2014.06.004.

Jain, Sonia, Cohen, Alison K. (2012). The Power of Developmental Assets in Building Behavioral Adjustment Among Youth Exposed to Community Violence: A Multidisciplinary Longitudinal Study of Resilience. Final Technical Report. NCJ 237915, San Francisco, CA: WestEd Health and Human Development Program [producer], National Institute of Justice [distributor].

Jennings, W. G., Piquero, A. R., \& Farrington, D. P. (2013). Does resting heart rate at age 18 distinguish general and violent offending up to age 50? Findings from the Cambridge Study in Delinquent Development. Journal of Criminal Justice, vol. 41, 213-219. DOI 10.1016/j.jcrimjus.2013.05.003.

Johansson, P., \& Kempf-Leonard, K. (2009). A Gender-Specific Pathway to Serious, Violent, and Chronic Offending? Exploring Howell's Risk Factors for Serious Delinquency. Crime \& Delinquency, Volume 55 Number 2, 216-240. DOI 10.1177/0011128708330652.

Kalvin, C. B., \& Bierman, K. L. (2017). Child and adolescent risk factors that differentially predict violent versus nonviolent crime. Aggressive Behavior, 43(6), 568-577. DOI 10.1002/ab.21715.

Katsiyannis, A., Thompson, M. P., Barrett, D. E., \& Kingree, J. B. (2012). School Predictors of Violent Criminality in Adulthood: Findings From a Nationally Representative Longitudinal Study. Remedial and Special Education, 34(4), 205-214. DOI $10.1177 / 0741932512448255$.

Kendler, K. S., Lonn, S. L., Morris, N. A., Sundquist, J., Langstrom, N., Sundquist, K. (2014). A Swedish national adoption study of criminality. Psychological Medicine, 44, 1913-1925. DOI 10.1017/S0033291713002638.

Khan, R., \& Cooke, D. J. (2008). Risk Factors for Severe Inter-Sibling Violence: A Preliminary Study of a Youth Forensic Sample. Journal of Interpersonal Violence, Volume 23, Number 11. 1513-1530. Doi 10.1177/0886260508314312. 
Knafo, A., Zahn-Waxler, C., Van Hulle, C., Robinson, J. L. (2008). The Developmental Origins of a Disposition Toward Empathy: Genetic and Environmental Contributions. Emotion, vol. 8, No. 6, 737-752. DOI 10.1016/S0140-6736(02)11133-0.

Krug, E. G., Dahlberg, T. T., Mercy, J. A., Zwi, A. B., \& Lozano, R. (2002). World report on violence and health. World Health Organization, Geneva. DOI $10.1177 / 1477370812467570$.

Lahlah, E., Knaap, L. M., \& Bogaerts, S. (2013). Dangerous boys or boys in danger? Examining the relationship between ethnicity, child abuse and violent offending. European Journal of Criminology, Vol. 10(6), 641-658. DOI 10.1177/1477370812467570.

Larson, M., Vaughn, M. G., Salas-Wright, C. P., \& Delisi, M. (2015). Narcisism, low selfcontrol, and violence among a nationally representative sample. Criminal Justice and Behavior, Vol 42, No. 6, 644-661. DOI 10.1177/0093854814553097.

Lee, S., Aos, S., Drake, E., Pennucci, A., Miller, M., \& Anderson, L. (2012). Return on investment: Evidence-based options to improve statewide outcomes. Olympia, WA: Washington State Institute for Public Policy.

Lipsey, M. W., \& Derzon, J. H. (1998). Predictors of serious delinquency in adolescence and early adulthood: A synthesis of longitudinal research. In Serious and Violent Juvenile Offenders: Risk Factors and Successful Interventions, edited by R. Loeber and D.P. Farrington. Thousand Oaks, CA: Sage Publications, Inc., 86-105.

Lundstrom, S., Forsman, M., Larsson, H., Kerekes, N., Serlachius, E. Langstrom, N., \& Lichtenstein, P. (2014). Childhood Neurodevelopmental Disorders and Violent Criminality: A Sibling Control Study. Journal of Autism Dev Disord, vol. 44, 2707-2716. DOI 10.1007/s10803-013-1873-0.

Moberg, T., Stenbacka, M., Tengstrom, A., Konsson, E. G., Nordstrom, P., Jokinen, J. (2015). Psychiatric and neurological disorders in late adolescence and risk of convictions for violent crime in men. BMC Psychiatry, 15:299.

Moya-Albiol, L., Herrero, N. y Bernal, M.C. (2010). Bases neuronales de la empatía". Revista de Neurología, 50 (2), pp. 89-100.

Muftic, L. R., Grubb, J. A., Bouffard, L. A., Maljevic, A. (2014). The Impact of Life Domains on Juvenile Offending in Bosnia and Herzegovina: Direct, Indirect, and Moderating Effects in Agnew's Integrated General Theory. Journal of Research in Crime and Delinquency, Vol. 51(6), pp. 816-845. DOI 10.1177/0022427814546198.

Muñoz, L. C., Frick, P. J., Kimonis, E. R., \& Aucoin, K. (2009). Verbal ability and delinquency: testing the moderating role of psychopathic traits. Journal of Child Psychology and Psychiatry 49:4, 414-421.

Nofziger, S., \& Kurtz, D. (2005). Violent Lives: A Lifestyle Model Linking Exposure to Violence to Juvenile Violent Offending. Journal of Research in Crime and Delinquency, vol. 42 no. $13-26$. DOI 10.1177/0022427803262061. 
Olate, R., Salas-Wright, C., \& Vaughn, M. G. (2012). Predictors of violence and delinquency among high risk youth and youth gang members in San Salvador, El Salvador. International Social Work, 55(3), 383-401. DOI 10.1177/0020872812437227.

Organização das Nações Unidas. (2015). Preventing Youth Violence: An Overview of the Evidence. Geneva, Switzerland: World Health Organization.

Pardini, D. A., Loeber, R., Farrington, D. P., Stouthamer-Loeber, M. (2012). Identifying Direct Protective Factors for Nonviolence. American Journal of Preventive Medicine, Vol 43. 28 40. DOI 10.1016/j.amepre.2012.04.024.

Piquero, A. R., Theobald, D., \& Farrington, D. P. (2014). The Overlap Between Offending Trajectories, Criminal Violence, and Intimate Partner Violence. International Journal of Offender Therapy and Comparative Criminology, Vol. 58(3), 286-302. DOI 10.1177/0306624X12472655.

Reingle, J. M., Jennings, W. G., \& Maldonado-Molina, M. M. (2012). Risk and Protective Factors for Trajectories of Violent Delinquency Among a Nationally Representative Sample of Early Adolescents. Youth Violence and Juvenile Justice, 000(00), 1-17. DOI $10.1177 / 1541204011431589$.

Reyes, J. W. (2007). Environmental Policy as Social Policy? The Impact of Childhood Lead Exposure on Crime.

Robbé, M. V., Vogel, V., \& Stam, J. (2012). Protective factors for violence risk: the value for clinica practice. Psychology. vol. 3, no. 12A, 1259-1263.DOI 10.4236/psych.2012.312A187.

Roberton, T., Daffern, M., \& Bucks, R. S. (2012). Emotion regulation and aggression. Aggression and Violent Behavior, 17(1), 72-82. Doi 10.1016/j.avb.2011.09.006.

Sariaslan, A., Larsson, H., D'Onofrio, B., Langstrom, N., \& Lichtenstein, P. (2014). Childhood family income, adolescent violent criminality and substance misuse: quasi-experimental total population study. The British Journal of Psychiatry, 205, 286-290. DOI 10.1192/bjp.bp.113.136200.

Schenker, M., \& Minayo, M. C. S. (2005). Fatores de risco e de proteção para o uso de drogas na adolescência. Ciência \& Saúde Coletiva, 10(3): 707:717.

Shetgiri, R., Boots, D. P., Lin, H., \& Cheng, T. L. (2016). Predictors of Weapon-Related Behaviors among African American, Latino, and White Youth. The Journal of Pediatrics, 171, 277-282. DOI 10.1016/j.jpeds.2015.12.008

Shoda, Y., Mischel, W. \& Peake, P. K. (1990). Predicting adolescent cognitive and selfregulatory competencies from preschool delay of gratification: Identifying diagnostic conditions. Developmental Psychology, Vol 26(6), 978-986. DOI 10.1037/00121649.26.6.978.

Silva, T. C., Larm, P., Vitaro, F., Tremblay, R. E., Hodgins, S. (2012). The association between maltreatment in childhood and criminal convictions to age 24: a prospective study of a 
community sample of males from disadvantaged neighbourhoods. Eur Child Adolesc Psychiatry, 21:403-413. DOI 10.1007/s00787-012-0281-x.

Smith, C. A., Ireland, T. O., Thornberry, T. P. (2005). Adolescent maltreatment and its impact on young adult antisocial behavior. Child Abuse Negl. Vol. 29 (10), 1099-1119. DOI 10.1016/j.chiabu.2005.02.011.

Souverein, F. A., Ward, C. L., Visser, I., \& Burton, P. (2015). Serious, Violent Young Offenders in South Africa. Journal of Interpersonal Violence, 31(10), 1859-1882. DOI $10.1177 / 0886260515570748$.

Tarullo, A. R., Obradovic, J., Gunnar, M. R. (2009). Self-Control and the Developing Brain. Zero to Three (J), v29 n3, 31-37.

Tremblay, R. E. (1999). When children's social development fails. In: Keating DP, Hertzman C, eds. Developmental health and the wealth of nations: Social, biological, and educational dynamics. New York: Guilford Press; 55-71.

Waiselfisz, J.J. (2014). O Mapa da Violência 2014: os jovens do Brasil. Secretaria Nacional de Juventude. Brasília.

Weijer, S. V., Jong, R. D., Bijleveld, C., Blokland, A., \& Raine, A. (2017). The Role of Heart Rate Levels in the Intergenerational Transmission of Crime. Societies, 7(3), 23. DOI $10.3390 /$ soc7030023.

Wikstrom, P.-O. H., \& Treiber, K. (2007). The role of self-control in crime causation. European Journal of Criminology, 4(2), 237-264. DOI 10.1177/1477370807074858.

\section{Os autores:}

André Vilela Komatsu é psicólogo e mestre em Psicologia pela Universidade de São Paulo (Ribeirão Preto). Atualmente cursa o Bacharelado em Estatística na Universidade de São Paulo (ICMC - São Carlos) e o Doutorado em Psicologia na área de Saúde e Desenvolvimento na Universidade de São Paulo (FFCLRP Ribeirão Preto).E-mail: avk@usp.br

Marina Rezende Bazon é docente do Departamento de Psicologia da Faculdade de Filosofia, Ciências e Letras de Ribeirão Preto da Universidade de São Paulo (USP-RP). Possui graduação em Psicologia pela Universidade de São Paulo (1991), Mestrado en Science - Psychoéducation pela Université de Montreal / Canadá (1995) e Doutorado em Psicologia pela Universidade de São Paulo (1999). E-mail: mbazon@ffclrp.usp.br.

Recebido em: 27/12/2017.

Aprovado em: 26/05/2018. 